\title{
GRAPHENE AS A QUANTUM PLAYGROUND
}

\author{
GIORGIO BENEDEK (*)
}

SUNTO. - Il moderno triathlon "calore-elettricitá-meccanica" ha un indiscutibile campione, il grafene, vantando esso, tra tutti i materiali in condizioni normali, record in tutte e tre le specialità: conduttivitá termica, mobilità elettrica e resistenza meccanica. D'altra parte il grafene, essendo perfettamente planare, è la forma più semplice delle possibili strutture sp2 di solo carbonio. La famiglia dei grafeni comprende forme curve come i fullereni, a curvatura gaussiana $G>0$ positiva, i nanotubi, a curvatura $G=0$ come il grafene, e le schwarziti a curvatura $G<0$, e curvatura media nulla. La coniugazione dei legami sp2 tra gli atomi di carbonio fa sì che molte proprietà elettroniche e vibrazionali globali dei grafeni dipendano primariamente dalla topologia della struttura. Esempi di proprietà globali stimate su base topologica sono il processo di crescita, la gerarchia degli isomeri, lo spettro vibrazionale, le costanti elastiche, la porosità in funzione dell'energia di deposizione, etc. La dinamica degli elettroni liberi nei grafeni è ben descritta dall'equazione quantistica relativistica di Dirac e diverse sue conseguenze come lo Zitterbewegung e il paradosso di Klein sono state dimostrate nel grafene. Pertanto il grafene permette di simulare e verificare teorie fondamentali della fisica delle particelle elementari e dell'astrofisica, assai meno accessibili agli esperimenti. Con qualche sorprendente previsione! Di fatto dalla fine '800 la topologia è diventata un paradigma di riferimento in molte branche della fisica fondamentale, dalla teoria topologica dell'elettricità di Hermann Weyl e dei wormholes cosmologici alla teoria delle stringhe e l'attuale teoria di campo topologica nella fisica delle alte energie.

$* * *$

ABSTRACT. - The modern triathlon "heat-electricity-mechanics" has an indisputable champion, graphene, as a recordman, among all materials in normal conditions, in all three specialties: thermal conductivity, electrical mobility and mechanical strength. On the other hand graphene, being perfectly planar, is the simplest of all possible sp2 pure carbon structures. The graphene family includes curved forms like fullerenes, having gaussian curvature $G>0$, nanotubes, with $G=0$ like graphene, and schwarzites with $G$

(*) Istituto Lombardo Accademia di Scienze e Lettere, Milano. Dipartimento di Scienza dei Materiali, Università di Milano-Bicocca, Milano, Italia. Donostia International Physics Center, Donostia/San Sebastian, Spain.

E-mail: giorgio.benedek@unimib.it 
$<0$ and vanishing mean curvature. The conjugation of carbon-carbon sp2 bonds makes several global electronic and vibrational properties of graphenes to primarily depend upon the structure topology. Global properties which can be estimated on topological grounds are the growth process, the isomer hierarchy, the vibrational spectrum, the elastic constants, the porosity as a function of the deposition energy, etc. The dynamics of free electrons in graphene is well described by the Dirac quantum-relativistic equation, and some of its consequences like the Zitterbewegung and Klein's paradox have been proved in graphene. Thus graphene allows for the simulation and validation of fundamental theories in fields hardy accessible to experiments like high-energy physics and cosmology. With some surprising prediction! It is a fact that since the late XIX century topology has become a reference paradigm in many branches of fundamental physics, from Hermann Weyl's topological theory of electricity and cosmological wormholes, to string theory and present topological field theories in high-energy physics.

\section{INTRODUCTION}

In recent years, after the work of Geim and Novoselov [1-3], the single layer of graphite has been promoted to the most important member of the graphene family. This family includes all pure $\mathrm{sp}^{2}$-bonded carbon structures and counts infinite members within each group defined by its topology [4] (Fig. 1). While graphite is known since antiquity, owing its name to traditional writing devices like charcoals and pencils, and its monolayers have been grown and studied on a large variety of substrates since half a century [5-8] only recently the infinite series of fullerenes, with the icosahedral $\mathrm{C}_{60}$ as the recognized leader $[9,10]$, has been welcomed into the family, followed by that, equally infinite, of carbon nanotubes [11], and, last but not least, of carbon schwarzites $[12-17,4]$.

The single layer of graphite, hereafter graphene par excellence, is actually a member of the group defined by a surface of topological genus $g=1$, which includes all nanotubes (Fig. 1c) and also toroidal (doughnutshaped) graphenes. The reason of such parenthood is that nanotubes and doughnuts are obtained by respectively rolling up once and twice a graphene sheet. Fullerenes have instead the topological genus of a sphere, $g=0$ (Fig. 1b), while integer genera larger than 1 include closed shapes with more than one handle (ring), as well as open surfaces (Fig. 1d) characterized by a negative Gauss curvature everywhere (schwarzites, from the name of the Swiss mathematician Helmut Schwarz [18] who developed the theory of tri-periodic minimal surfaces). The toy basket also includes possible graphene of any half-integer genus represented by single-face surfaces like Möbius rings and Klein bottles. Although integer 
and half-integer genus surfaces have the same dignity in topology as have particles with integer (bosons) and half-integer spin (fermions) in quantum physics, our playground shall be restricted to graphenes with integer genus, leaving to chemists the pleasure, actually a difficult task) of synthesizing new single-face graphene forms.
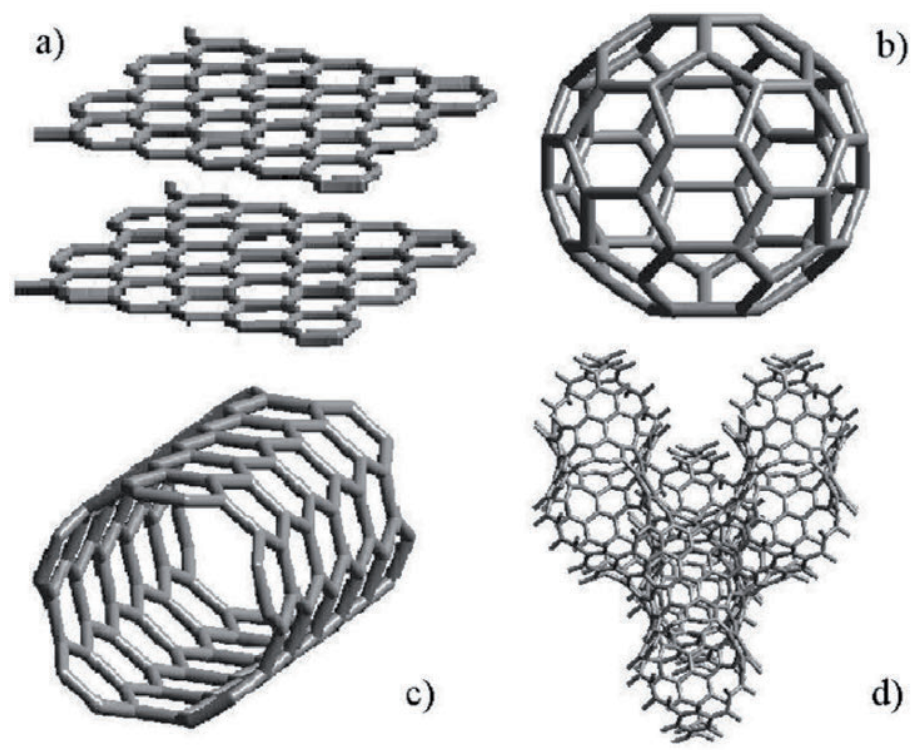

Fig. 1. The four canonical $s p^{2}$ carbon forms grouped under the general name of graphenes. a) Graphite; the graphite monolayer is currently known as graphene. (b) Fullerene; the picture shows the most popular of all fullerenes, the icosahedral $C_{60}$. (c) Single-walled nanotube. (d) A three-periodic schwarzite.

\section{THE WIZARDY OF CONJUGATION}

Graphene, by combining its superior electrical and thermal conductivities with the highest tensile strength, represents an ideal triad, fully in the spirit of the present conference. The bond conjugation which characterizes fully $\mathrm{sp}^{2}$-carbon structure constitutes a holistic property, in the sense that imposing the character of a single bond to be double or single can determine the bond character in the whole structure at any distance and almost instantaneously, the speed of propagation of the signal being expressed by the Fermi velocity. Such an infinite-range interaction makes topology the leading property over local metric properties. 
Consider the small portion of graphene depicted in Fig. 2a, where grey bonds are single and red bonds are double. You may exchange them arbitrarily provided each atom links three other atoms with four (one red and two grey) bonds. In quantum mechanics each configuration fulfilling the above rule is possible with a given probability, and chosing one or another in a graphical representatio is just a matter of taste. The correlation between neighboring bonds which extends all over the surface however extended is the essence of conjugation. Imagine now that you wish to roll up the portion of graphene (a) to make a nanotube, by joining either the two vertical edges or the two horizontal edges: since there is an exact correspondence of the red and grey bonds in both the vertical and horizontal edge pairs, the nanotube can be made in both directions, and also the doughnut can be made by joining both edge pairs. The configuration (b), where the four single bonds in the yellow rectangle of (a) have been turned into double bonds, is also plausible for the graphene portion but cannot form a nanotube by joining the vertical edges because double bonds are requested on the left and single bonds on the right side. No problem for the horizontal nanotube, and also for the vertical one it is possible to reshuffle the bonds so has to have the same set of double bonds in the yellow strip. The example above allows however to conclude that the cyclic boundary conditions which are imposed in order to roll up (close) the sheet reduces the number of possible bonding configurations.
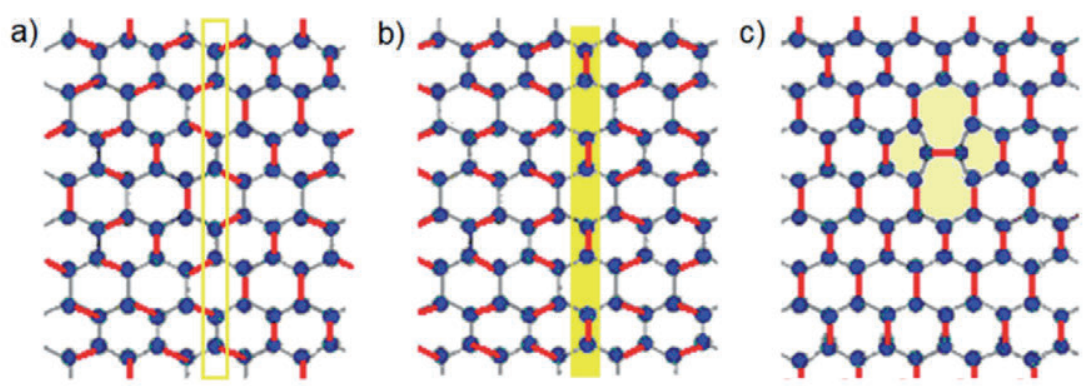

Fig. 2. (a) A possible configuration of single (thin segment) and double (thick red segment) bonds in a portion of graphene fulfilling cyclic boundary conditions. Any other disposition of bonds is equally possible provided each atom forms one double bond and two single bonds and cyclic boundary conditions are fulfilled. (b) In this bond configuration the vertical edges do not fulfill the cyclic boundary condition.

(c) A Stone-Wales defect where four adjacent 6-fold rings have been turned into a pair of 5-fold and a pair of 7-fold rings. 
The bonding rule of graphenes (coordination 3 with one double and two single bonds for each atom) is an example of topological rule. There are many ways to dispose the atoms so as to fulfill this rule: Fig. $2 c$ shows a typical topological defect, the Stone-Wales transformation in which one of the bonds within the yellow strip in (b) has been rotated by $90^{\circ}$ so as to transform four adjacent hexagons into a pair of pentagons and a pair of heptagons. The pairs of horizontal and vertical boundaries are now perfectly coherent so that the Stone-Wales defect may occur in nanotubes as well as in doughnuts. Actually it is a basic defect in all graphene families, I would say a familiar defect.

In general any graphene of the family can be viewed as a tesselation of some surface of genus $g$ with a given number $v$ of atoms (nodes), a given number $e$ of bonds (edges) and a given number $f$ of rings (polygonal faces or tiles). These numbers are not completely independent: they have to fulfill a famous theorem due to Euler, stating that

$$
\begin{aligned}
v-e+f & =\chi \\
& =3-k=2(1-g)
\end{aligned}
$$

where $\chi$ is the Euler-Poincaré characteristic. This topological constant is related to genus via the third equality of (1) and to the Hilbert connectivity $k$. The connectivity of a surface $k$ is an integer positive number with an interesting definition: the maximum number of close cuts which can be made on a surface without splitting it apart plus one. For example a circular cut on a plane (the projective plane, including one point at infinity) or a sphere is always enough to separate a circle from the rest: thus $k=1$; in a Möbius ring we can make a close longitudinal cut which however does not split the ring into two parts; another close cut is needed to split it apart: thus $k=2$. A torus (as well as a plane with two cyclic boundary conditions) can stand two close cuts without splitting into two parts: a third one is necessary and therefore $k=3$, etc. Odd (even) values of $k$ define two-face (singleface) surfaces. As a consequence spheres and projective planes have $\chi=2, g=0$; the Möbius ring $\chi=1, g=1 / 2$; the torus (or a plane with two cyclic boundary conditions, or a nanotube with one cyclic boundary condition) $\chi=0, g=1$; the Klein bottle $\chi=-1, g={ }^{3} / 2$; the two-hole torus $\chi=-2, g=2$, etc. Thus the genus $g$ is equal to the number of holes of a multi-hole torus).

For a graphene fulfilling the three-fold coordination rule it is 
$e=\frac{3}{2} v, \quad \frac{1}{2} v=f-\chi$.

Since both $f$ and $\chi$ are integer numbers the number of atoms of a closed graphene (e.g., a fullerene) is always even. For periodic schwarzites it may be convenient to refer all quantities in Eq. (1) to a unit cell and to close it on itself by imposing cyclic boundary conditions. Each closure creates a torus hole and therefore the unit element of a schwarzite with periodic boundary condition shall always be characterized by a positive, possibly large value of $g$.

The Euler theorem yields also a rule on the distribution of polygons tiling the surface of graphenes: by calling $f_{n}$ the number of polygons ( $n$-membered rings), so that $f=\Sigma_{n} f_{n}, v=\Sigma_{n}(n / 3) f_{n}$, Eq. (2) gives

$$
6 \chi=\sum_{n}(6-n) f_{n}
$$

Note that this conditions on the numbers of different rings is independent of the number of 6-rings, which can therefore be any natural number, including 0 and except 1 . Hereafter we shall consider only the most frequent graphenes, i.e., those with six-, five- and seven-membered rings, the extension to other possible structures with larger or smaller rings being straightforward. With such restriction it is found that

$$
f_{7}-f_{5}=-6 \chi \text {. }
$$

For fullerenes $(\chi=2)$ with no 7-rings the well known result $f_{5}=12$ is obtained. For open nanotubes and graphene sheets $f_{5}=f_{7}$ : in perfect structures this number can be zero, whereas in defective graphene or nanotubes 5 - and 7 -rings always occurs in pairs, e.g., through the StoneWales transformation which converts four adjacent 6-rings into two 5 7 ring pairs (Fig. 2c).

The simplest forms of periodic schwarzites have a unit coordinating either six identical units through six necks in the three orthogonal direction, thus forming a simple cubic lattice (P-type schwarzites), or four identical units through four necks in the tetrahedral direction, thus forming a diamond lattice, i.e., a face-centered cubic lattice with two units per unit cell (D-type schwarzites). Fig. $1 d$ depicts a portion of a D-type schwarzite. In both P- and D-type schwarzites, the unit cell is connected to the six neighboring cells via six necks, which is equivalent to say that the unit cell of both forms can be closed on itself by fulfilling three cyclic boundary conditions, i.e., via three torus rings: thus $g=3$, 
or $\chi=-4$ per unit cell, and therefore in the absence of pentagons, Eq. (4) requires 24 heptagons/cell, whatever is the number $(\neq 1)$ of hexagons, and each tetrahedral element of a D-type schwarzite has $f_{7=12}$. The smallest D-type schwarzite with no hexagons has 28 atoms per element (56 per unit cell, like the smallest P-type schwarzite), whereas the smallest fullerene $\left(f_{5}=12, f_{7}=0\right)$ is $C_{20}$, having $(12 \times 7-12 \times 5) / 3=8$ atoms less the the unit element of the smallest D-type schwarzite. In conclusion the family of D-type schwarzites has the formula $f_{c c}-\left(\mathrm{C}_{k}\right)_{2}$ where $k=28+2 f_{6}$ is the number of atoms per element, while the simple-cubic P-type schwarzites are denoted by $s c-\mathrm{C}_{m}$ with $m=56+2 f_{6}$.

The Euler theorem has also practical consequences in Nature: the famous naturalist and philosopher Ernst Haeckel baptized a beautiful radiolarian, whose skeleton is shown in Fig. 3, Aulonia bexagonia, without considering that a hexagonal tiling cannot be closed on a sphere without adding 12 pentagonal tiles, or 14 pentagonal and 2 heptagonal tiles, etc. (e.g., by allowing for Stone-Wales defects: the reader can easily find 5- and 7 -membered rings). Of course, amid such a large number of hexagons, a few pentagons and even less heptagons are negligible, though it would be a mistake to present them as errors of nature!

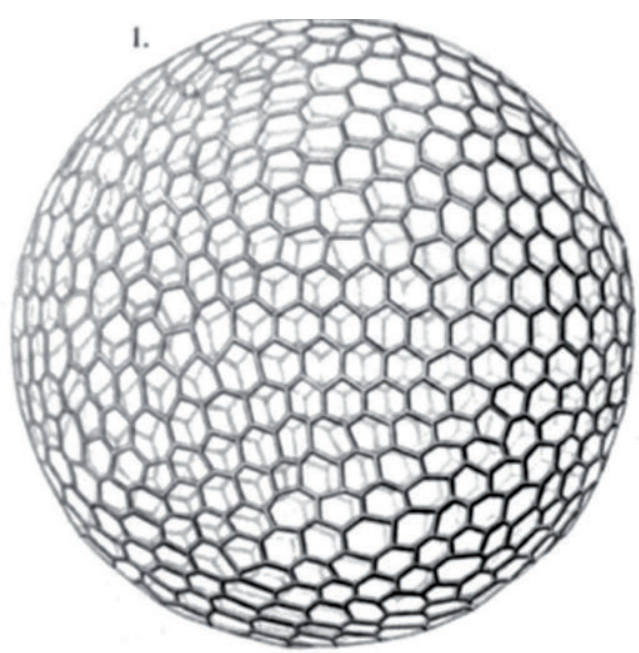

Fig. 3. Aulonia hexagonia, Heckel [19]. Despite the given name of this radiolarian, a sphere cannot be floored with exclusively hexagonal tiles. At least four triangular, or six square, or twelve pentagonal tiles need to be added in order to complete the work (cfr. Eq. 3). The reader can easily find several non-hexagonal tiles. 


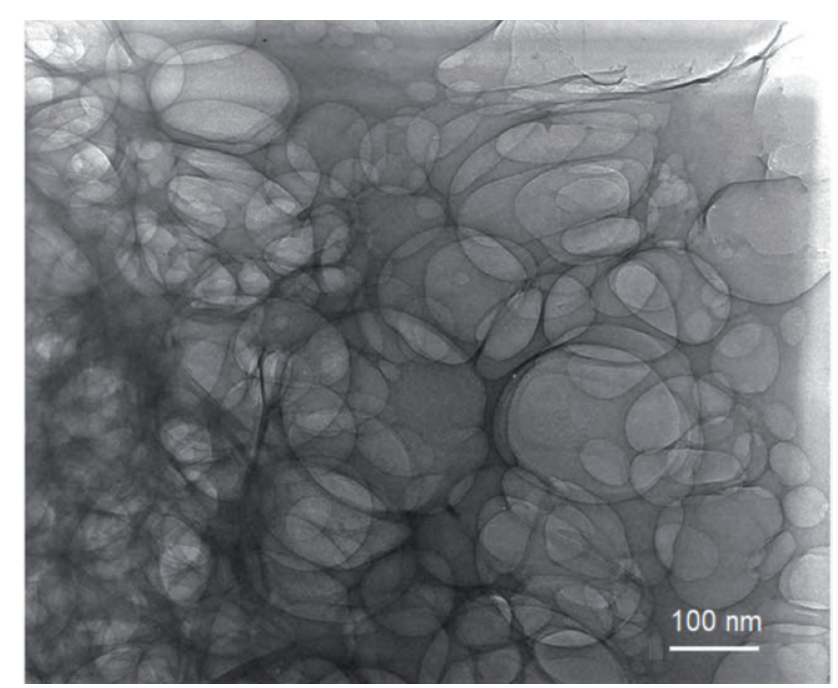

Fig. 4. A transmission electron microscopy (TEM) image of a random carbon schwarzite. The structure consists in a continuous, presumably minimal surface extending in three dimensions ans held together by graphitic sp $p^{2}$ bonds $[15,4]$.

In general it appears that graphenes with a dominant positive Gauss curvature $(G>0)$ like fullerenes have more pentagons than heptagons, while those with a dominant negative Gauss curvature $(G<0)$ have more heptagons than pentagons. Moreover abutting pentagons are energetically unfavored while heptagons tend to coalesce. The smallest fullerene with no abutting pentagons, the $\mathrm{C}_{60}$ isomer of icosahedral symmetry, is actually the most stable and therefore the most abundant in carbon cluster beams. On the contrary the schwarzites with the highest point-symmetry are unfavored with respect to isomers with abutting heptagons. This is apparently the reason why the attempts to grow periodic schwarzites by means of supersonic cluster beam deposition (SCBD) have been so far unsuccessful [4,14-16]. Nevertheless the SCBD of carbon clusters seeded with catalist nanoparticles [15] yields virtually pure $\mathrm{sp}^{2}$ random schwarzites (Fig. 4) with a very high specific surface and a porosity strongly dependent on the average deposition energy, in agreement with previous molecular dynamics simulations [14]. Despite the apparent randomness of the structure as appears from TEM images (Figs. 4 and 5b), an analytical simulation reveals a fractal self-affine structure growing from a catalyst nanoparticle $[4,15,17]$. The ultra-high porosity of carbon schwarzites and their relative chemical 
inertness has already enabled various nanotechnologies [17,20,21], almost two decades before the same structures have been glamorously proposed under the new name of 3D graphene [22].

a)

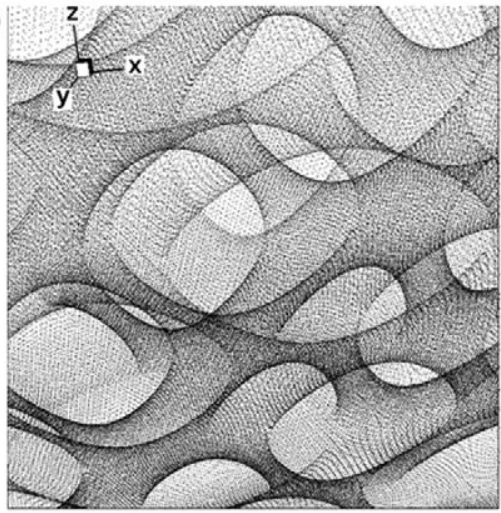

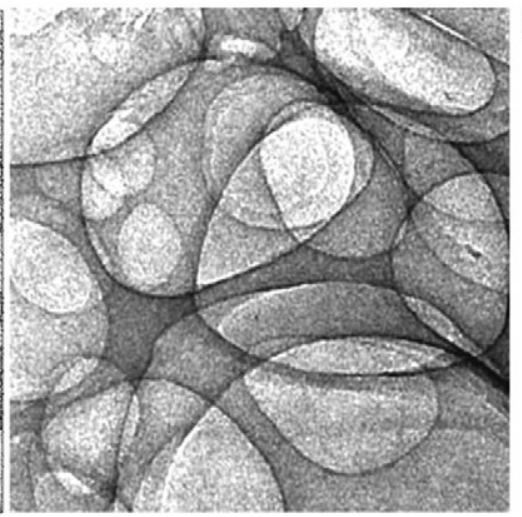

Fig. 5. The simulation of the TEM images of carbon schwarzites with a self-affine fractal minimal surface growing in the z-direction (a) provides a faithful visual representation of experimental TEM pictures (b) $[15,23]$.

\section{Where Quantum Physics Meets Topology}

A natural question posed by SCBD growth of graphene structures is whether, depending on various experimental conditions like substrate structure, composition and temperature, catalyst, beam energy, etc., fullerenes or nanotubes or schwarzites, or just trivial graphite monolayers will be produced. Of course many other exotic, $\mathrm{sp}^{2}-\mathrm{sp}^{3}$ mixed structures are around the corner. This essentially and ultimately depends on thermodynamic conditions, more specifically (at equilibrium) on extensive properties such as the free energy of the different forms. Topology provides in this case a dramatic simplification through the wonderful Gauss-Bonnet theorem which links local properties, represented by the Gauss curvature $G$, to a global property, the topological Euler-Poincarè characteristic $\chi$. The theorem states that:

$$
\int_{S} G d S=2 \pi \chi
$$

where $\mathrm{d} S$ is the surface element, and the integral extends over the entire surface, supposed to be closed as explained above. The total energy of 
a curved single-walled graphene can be expressed in the form suggested by Helfrich for membranes and foams $[23,24-26]$ :

$$
\begin{aligned}
E & =\int_{S} d S\left(\gamma+\kappa H^{2}-G\right) \\
& =-2 \pi \chi \bar{\kappa}+S\left(\gamma+\kappa<H^{2}>\right)
\end{aligned}
$$

where $\gamma=2.82 \mathrm{eV} / \AA^{2}$ is the energy for unitary flat surface (a graphite sheet) [27], $H$ the mean curvature and $\left\langle H^{2}>\right.$ the average of its square over the surface. The constants $\kappa$ and $\bar{\kappa}$ are two elastic constants associated with cylindrical and elliptical/hyperbolic deformations of the surface, respectively. For minimal surfaces like schwarzites $H=0$ everywhere, and therefore the difference $E-S \gamma=-2 \pi \chi \bar{\kappa}>0$, expressing the energy required to fold a graphene sheet into a schwarzite (which is positive since $\chi<0$ ) depends exclusively on the surface topology and on the actual value of $\bar{\kappa}$, which is assumed to be a constant independent of the position on the surface.

Density functional (DF) calculations in the local density approximation for nanotubes of variable radius $R$ (where $H=1 /(2 R)$ and $G=0$ ) $[27,28]$ and $C_{60}$ (where $H=1 / R$ and $G=1 / R^{2}$ ) [27] permit to extract the values $\kappa=3.1 \mathrm{eV}$ and $\bar{\kappa}=1.7 \mathrm{eV}$. Similar values (within $\sim 5 \%$ ) are extracted from quantum tight-binding calculations for schwarzites [29], which proves an approximate universality of the Helfrich total energy for the whole family of single-walled graphenes. It should be noted that Eq. (6) is actually an expansion into curvatures of increasing order truncated at the first three terms.

It is now important to observe that the local initial values of $\bar{\kappa}$ and $\kappa$ either at the surface termination into vacuum, where the growth takes place by cluster addition, or at the contact with the substrate or the catalyst, are likely to be quite different from the above values (derived for free-standing closed structures) and should be obtained from ab-initio calculations. In particular the local change in the electronic structure, e.g., a $\pi$ bond-charge depletion or accretion, can substantially modify $\bar{\kappa}$. The charge redistribution produced by a catalyst depends on the actual size of catalyst nanoparticles, which may explain why in presence of very small and highly dispersed metallorganic precursors the growth of schwarzites supersedes that of nanotubes. The initial relative values of $\kappa$ and $\bar{\kappa}$ which determine whether the growth process of $\mathrm{sp}^{2}$ carbon preferentially leads to fullerenes, nanotubes or schwarzites, define the three topological domains shown in Fig. $6[17,23]$ : schwarzites are favoured for $\bar{\kappa}<1 / 4 \kappa$, nanotubes for $1 / 4 \kappa<\bar{\kappa}<3 / 4 \kappa$ and fullerenes for $\bar{\kappa}>3 / 4 \kappa$. 
When $\bar{\kappa} \approx \kappa / 2$, as found from DFT calculations for free-standing graphenes, nanotubes are more likely to occur.

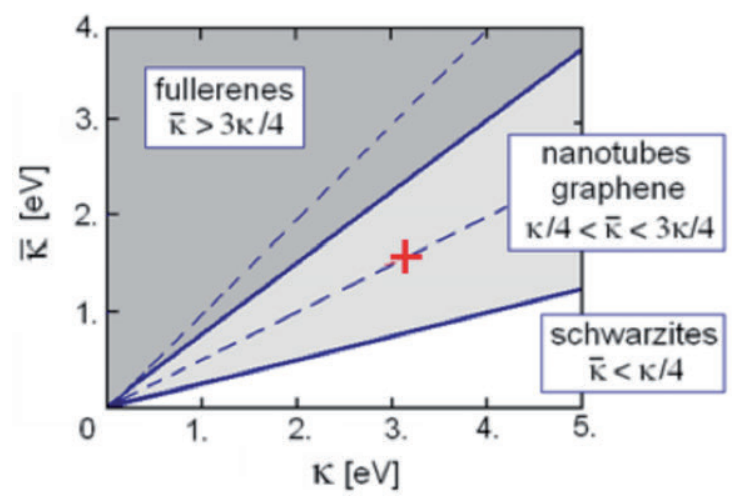

Fig. 6. Topological domains for the growth of $s p^{2}$ carbon graphenic structures as functions of the incipient elastic constants. The $\bar{\kappa}$ and $\mathrm{\kappa}$ values

for free-standing graphenes (red cross) favour the growth of nanotubes [16,23].

Once the growth process has started, the local elastic constants $\bar{\kappa}$ and $\kappa$ away from the initial conditions tend to their universal values, but the growth proceeds within its topological basin, since switching to another topology would require a massive reshuffling of bonds. This is an example of topological robustness, which guides the growth process along of a quasi-deterministic path.

We have considered so far the topological consequences arising from the tesselation of surfaces of different genus with 6-, 5- and 7 membered carbon rings as an effect of that peculiar quantum property of $\mathrm{sp}^{2}$ bonding which we call conjugation. Also graph topology bears interesting implications and simplifications in the theory of $\mathrm{sp}^{2}$ carbon conjugated systems. The main character in graph theory is the adjacency matrix A whose elements $A_{i j}$, are 1 for all pairs of carbon atoms $i$ and $j$ which are connected by a bond, and 0 for all the other pairs $(i, j)$, including $i=j$. The electronic states of a graphenic structure of any topology can be estimated within a linear combination of atomic orbital (LCAO) scheme by solving the system

$(\mathrm{I}-s \mathrm{~A})^{-1}(\beta \mathrm{A}+\alpha \mathrm{I}) \mathbf{c}=E \mathrm{I} \mathbf{c}$,

where $\mathrm{I}$ is the identity matrix, $E$ an eigenvalue and $\mathbf{c}$ the corresponding eigenvector providing the LCAO coefficients. The diagonalization of Eq. 
(7) with the LCAO matrix elements $\alpha=-2.60 \mathrm{eV}$ and $\beta=3.01 \mathrm{eV}$ and overlap $s=0$ (Hückel approximation) [30] provides for the fullerene $\mathrm{C}_{60}$ a set of eigenvalues (Fig. 7, lower abscissa in $\mathrm{eV}$ ) with the respective density of states (DOS, shadowed peaks for occupied states) in excellent agreement with the Hückel eigenvalues reported by Bühl and Hirsch (upper abscissa, in units of $\beta$ ) [31]. It will be appreciated that the adjacency matrix only contains topological data with no information on the structural details. On this basis it is expected that the diagonalization of the adjacency matrix for other graphenic topologies with the above parameters should as well provide a first-hand estimation of the valence electron energy levels.

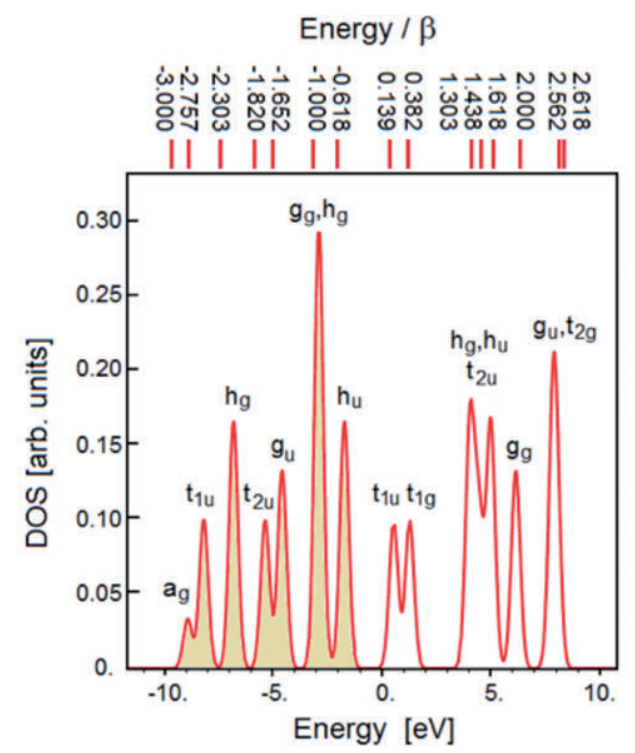

Fig. 7. Topological electronic states. The density of states (DOS, with shadowed peaks corresponding to occupied states) associated with the energy levels (in $\mathrm{eV}$ ) of icosahedral $C_{60}$ with their respective irreducible representation labels as obtained from the diagonalization of the adjacency matrix with $\alpha=-2.60 \mathrm{eV}$ and $\beta=3.01 \mathrm{eV}$ and overlap $\mathrm{s}=0$ (Hückel approximation) [30], compared with the Hückel energy levels (in units of $\beta$ ) reported by Bübl and Hirsch [31].

A similar topological approach works for the calculation of phonon states. In this case the eigenvalue equation providing the angular frequencies $\omega=\omega_{\alpha v}$ and the components $u_{i}=u_{i \alpha, v}$ of the atomic displacements for each phonon $v$ and each polarization $\alpha=\perp, \|$ can be expressed in terms of the adjacency matrix as 
$-M \omega^{2} u_{i}=f_{\alpha} \sum_{j}\left(A_{i j}-3 \delta_{i j}\right) u_{j}, \quad \alpha=\perp, \|$,

where $M$ is the carbon atom mass, and the term with the Kronecker delta is implied by the translational invariance of the system hamiltonian. The force constants $f_{\alpha}$ can be fitted to the respective frequencies $\omega_{\perp}$ and $\omega_{\|}$for graphene and considered to be transferable to other $\mathrm{sp}^{2}$ carbon structures. The angular frequencies can then be expressed in terms of the eigenvalues $\lambda_{\alpha v}$ of the adjacency matrix as

$\omega_{\alpha v}=\left[\frac{f_{\alpha}}{M}\left(3-\lambda_{\alpha v}\right)\right]^{1 / 2}$.

An example of topological phonon spectrum calculated with Eqs. $(8,9)$ is shown in Fig. 8 for the icosahedral fullerene $\mathrm{C}_{60}[30,32]$ and compared with the experimental spectrum obtained with inelastic neutron scattering (NIS) [33]. The good correspondence of the NIS spectrum peaks to those of the topological phonon spectrum indicates that the gross features of the $\mathrm{C}_{60}$ vibrational spectrum are accounted for by its topology, i.e., by its bonding network. Similar results have been obtained for the D-type schwarzite $\left(\mathrm{C}_{28}\right)_{2}$ for which a comparison is possible between the ab-initio and the topological phonon spectra [30].

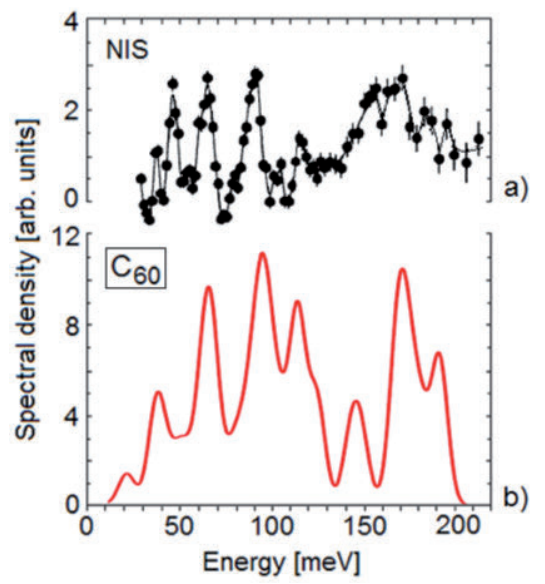

Fig. 8. Comparison between the vibrational spectrum of the icosabedral fullerene $C_{60}$ measured with neutron inelastic scattering (NIS) [33] (a), and the topological phonon spectrum calculated from the adjacency matrix (b) [30,32].

Topological phonon spectra cannot of course be as accurate as those obtained from detailed lattice dynamics calculations, but may be 
conveniently used in the calculation of integral properties such as, e.g., the vibrational part of thermodynamic functions. As an example, it is perfectly sufficient to use the topological phonon frequencies for the calculation of the mean-square atomic displacement relative to the interatomic distance $d$, given by the equation

$$
v(T) \equiv \frac{1}{d}\left\langle u^{2}\right\rangle_{T}^{1 / 2}=\frac{1}{d}\left[\sum_{\lambda} \frac{\mathrm{h}}{2 N M \omega_{\lambda}}\left(\frac{1}{e^{\mathrm{h} \omega_{\lambda} / k T}-1}+\frac{1}{2}\right)\right]^{1 / 2},
$$

It is possible to estimate the temperature at which the bonds start breaking leading to melting by means of the Lindemann criterion: for carbon materials this occurs at $v\left(T_{m}\right)=0.084$ [34]. A semi-empirical tight-binding molecular dynamics simulation of the topological connectivity as a function of temperature for the D-type schwarzite fcc- $\left(\mathrm{C}_{36}\right)_{2}$ (Fig. 9a) [35] shows that a graphitization transition, consequent to a rapid break of prevalently single bonds, is predicted to occur around $4000 \mathrm{~K}$. At this temperature $T_{g}$ the ratio $v\left(T_{g}\right)$ derived from Eq. (10), with the topological phonon spectrum (Fig. 9b) and the graphite interatomic distance $d=1.42 \AA$, is equal to 0.077 . Raising the temperature beyond graphitization, melting of graphite sheets occurs.
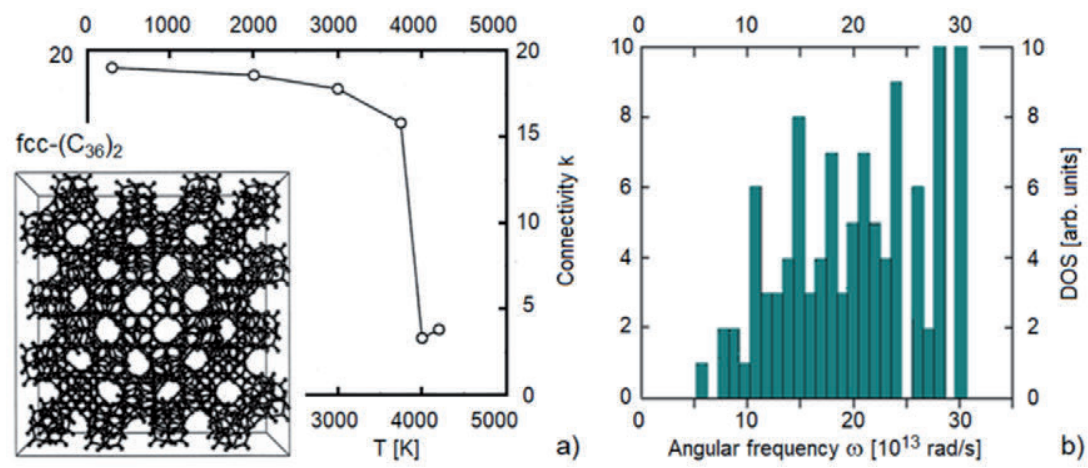

Fig. 9. (a) The three-dimensional fcc lattice generated by the schwarzite $f_{c c}-\left(C_{30}\right)_{2}$ represented within a simulation cube containing $8 C_{36}$ elements ( $4 f_{c c}$ unit cells).

On the background the evolution of the topological connectivity as function of temperature from the low temperature value $k=19$ for the schwarzite within the simulation cube to the high-temperature value $k=3$ for graphite. This molecular dynamics simulation predicts the topological transition to graphite (graphitization) at $4000 \mathrm{~K}$ transition [35]. (b) The topological phonon distribution at zero for a single element $C_{36}$ using adjacency matrix diagonalization. 
A simulation for graphene by to Zakharchenko et al [36] gives a melting temperature of $4900 \mathrm{~K}$, which would correspond, on the basis of Eq. (10), to $v\left(T_{m}\right)=0.085$, in good agreement with the Lindemann criterion for carbon materials. In view of such a good correspondence for melting, this analysis provides therefore a criterion for the graphitization transition of schwarzite structures, which can be confidently fixed at $v\left(T_{g}\right)=0.077$. Fig. $9 a$ offers a clear example of a phase transition with a topological index acting as an order parameter.

The assumption that the total energy of graphenes can be assimilated to that of a continuous elastic membrane in the Helfrich's form, Eq. (6), is a crude approximation, which does not permit, for example, to distinguish among isomers, not even those generated by StoneWales defects or simply redistributing the same carbon rings. For schwarzite it seems reasonable to assume that minimality is best approached by the most stable isomer, but less stable isomers deviate more and more from the minimality conditions, acquiring a non-zero mean curvature. This is a clear effect of the non-continuous, tesselated structure of graphenes, and it is graph topology which provides the next refinement.

A closed graphene can be viewed as a graph with $N$ nodes (carbon atoms). By defining the topological distance between two nodes as given by the minimum number of steps (bonds) which connect the two nodes, the graph can be considered as made of $s$ atomic shells, each shell including the $s_{j}$ atoms which have the same topological distance $j$ from an atom taken as origin $(j=0)$. Since the maximum topological distance normally depends on the choice of the origin atom, $s$ is defined as the maximum topological distance in the given graph. With these definitions a sum over the atom index $m$ from 0 to $N$ - 1 is replaced by a sum over the $s_{j}$ atoms belonging to the $j$-th shell times the sum over the $s+1$ shells (including the origin, $j=0, s_{0}=1$ ). The local Wiener index of order $n$ referred to the site 0 is then defined as

$$
w_{0}^{(n)} \equiv \frac{1}{n !} \sum_{j=0}^{s} j^{n} s_{j}
$$

It was recently demonstrated [30] that the LCAO binding energy of any graphene atom taken as origin is proportional to an expansion over its local Wiener indices of increasing order and that, for sufficiently large $N$, truncation to order 1 is generally a good approximation. The total $1^{\text {st }}$ order Wiener index 


$$
W^{(1)}=\frac{1}{2} \sum_{h=0}^{N-1} w_{h}^{(1)}
$$

turns out to be approximately proportional to the total energy, as Harry Wiener conjectured long ago about the boiling points of paraffins [37]. Thus the Wiener index is shown to provide a reasonable ranking of the isomers according to their stability, working de facto as a sort of configurational potential [30]. This finding extends to all graphenes, as anticipated by Ori et al in a seminal study on $\mathrm{C}_{66}$ fullerene isomers [38].

Tab. 1. The 21 isomers of the $\mathrm{C}_{36}$ element of the fcc- $\left(\mathrm{C}_{36}\right)_{2}$ schwarzite ordered according to the increasing Wiener index $W^{(1)}$, with the indication of their point symmetry group. The most stable isomer is not that of the highest point symmetry $\mathrm{T}_{2}$, (shown in Fig. 9a), but the one of point summetry $\mathrm{C}_{2 \mathrm{v}}$ [30].

\begin{tabular}{llllll}
\hline Isomer & $\mathbf{S y m}$ & $\mathbf{W}^{(1)}$ & 11 & $\mathrm{C}_{2}$ & 4918 \\
\hline 1 & $\mathrm{C}_{2 \mathrm{v}}$ & 4839 & 12 & $\mathrm{C}_{1}$ & 4954 \\
\hline 2 & $\mathrm{C}_{2}$ & 4846 & 13 & $\mathrm{C}_{2}$ & 4966 \\
\hline 3 & $\mathrm{D}_{2}$ & 4848 & 14 & $\mathrm{C}_{1}$ & 4993 \\
\hline 4 & $\mathrm{C}_{\mathrm{S}}$ & 4854 & 15 & $\mathrm{C}_{2}$ & 5020 \\
\hline 5 & $\mathrm{D}_{2 \mathrm{~d}}$ & 4856 & 16 & $\mathrm{D}_{2}$ & 5046 \\
\hline 6 & $\mathrm{C}_{2}$ & 4875 & 17 & $\mathrm{~S}_{4}$ & 5046 \\
\hline 8 & $\mathrm{C}_{1}$ & 4887 & 18 & $\mathrm{C}_{2}$ & 5049 \\
\hline 9 & $\mathrm{C}_{1}$ & 4891 & 19 & $\mathrm{~T}_{\mathrm{d}}$ & 5214 \\
\hline 10 & $\mathrm{C}_{1}$ & 4897 & 20 & $\mathrm{C}_{2}$ & 5399 \\
\hline
\end{tabular}

Tab. 1 lists the 21 isomers with only 6- and 7-membered rings of the $\mathrm{C}_{36}$ element of the fcc- $\left(\mathrm{C}_{36}\right)_{2}$ schwarzite ordered according to the increasing Wiener index $W^{(1)}$, i.e., from the most to the least stable. As appears from the respective point symmetry group shown in the second column, the most stable isomer is not the one with the highest (tetrahedral) point symmetry $\mathrm{T}_{2}$, shown in Fig. 9a, but the one of point symmetry $C_{2 v}$ [30]. This is apparently opposite to the behaviour of fullerene isomers, where the most symmetric are also the most stable 
(e.g., the icosahedral $\mathrm{C}_{60}$ ), and is likely to be related to the fact mentioned above that the twelve 7 -membered rings of each schwarzite element tend to dispose themselves as much as possible one next to the other, whereas the twelve 5-membered rings of a fullerene tend to stay apart. While the latter trend is compatible with the higher symmetries, the former favours lower symmetries. This is probably the reason why the SCBD growth of well ordered periodic schwarzites was not so far succesful, the amourphous self-affine structures being the natural output in both quantum molecular dynamics simulations [14] and experiment [15].

\section{From Graphenes to Other Playgrounds [39]}

The mathematical theory of minimal surfaces quickly impacted physics. A first, famous example is the work of Joseph Plateau on soap foams (Fig. 10). Plateau's pioneering work and the laws bearing his name [40], received a complete mathematical demonstration only 40 years ago by Jean Ellen Taylor [41], but the beautiful partitions of the three-dimensional space into volumes, separated by minimal surfaces so as to minimize the thermodynamic potentials, inspired new theories and models. For example Lord Kelvin conjectured that equal polyhedra having the shape of the cubo-octahedron provide a "division of space with minimum partitional area" [42]. In other words they would be the ideal shape of milk containers which could be perfectly packed with no waste of expensive covering and space. Actually better solutions have been found than Kelvin's with different polyhedra of equal volume, clathrates (Fig. 10a) - e.g., the celebrated Weaire-Phelan structure [43] - which left then the realm of topology for that of crystallography. Topology became soon a reference paradigm in many branches of fundamental physics, besides schwarzites (Fig. 10b), which range from Hermann Weyl's topological theory of electricity and cosmological wormholes [44], to string theory Dbranes [45], and present topological field theories in high-energy physics [46]. The physics of condensed matter is to a great deal that of its electrons and much depends on the topology of the Fermi surface and of the electron energy bands. The equilibrium structure of the solid and its phase diagram crucially depend on these electronic features, and so do quantum phase transitions. 


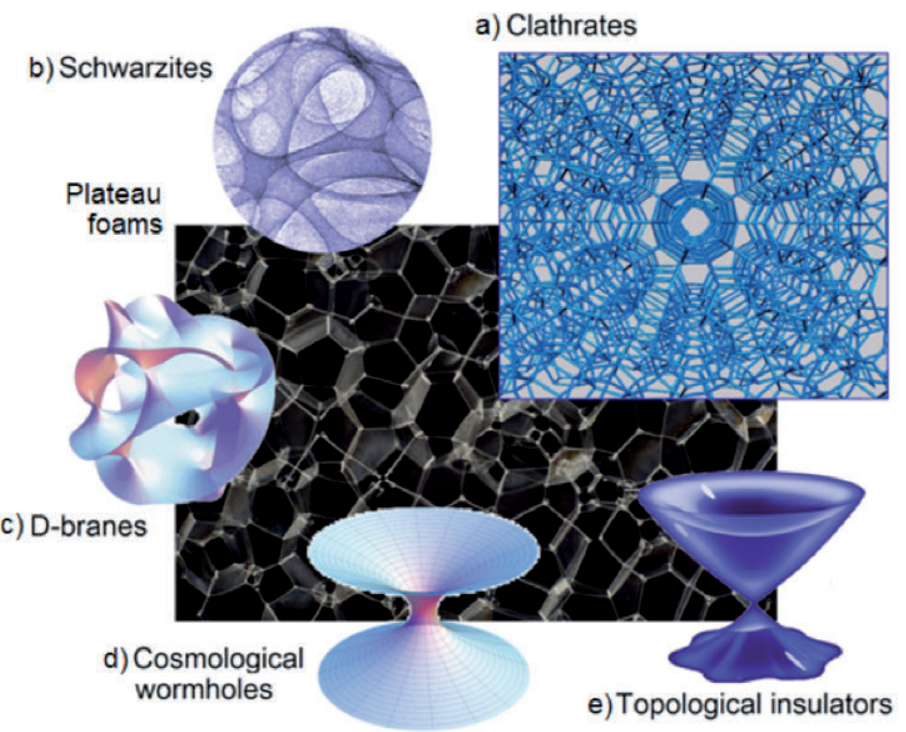

Fig. 10. On a soap foam background organized according to Plateau's laws five topological structures representing different physical systems are displayed:

(a) a bex-Si $i_{40}$ clathrate filling the space with polybedra similarly to a soap foam [47]

(b) a transmission electron microscope image of spongy carbon with the structure of a self-affine minimal schwarzite [15] (c) cross section of the quintic Calabi-Yau manifold representing a D-brane, produced using the methods described in [48] (image from Wikipedia Commons); (d) a representation of a Schwarzschild wormbole connecting two distant regions of the space-time [49]; (e) similar topological features may be found in the surface band structures of a topological insulators, however with the neck reduced to a point, termed as Dirac point [50].

In the early seventies Vadim Berezinskii, an Ukrainian physicist working at the Landau Institute in Moscow, and the two British physicists Michael Kosterlitz and David Thouless approached independently the problem of phase transitions in two-dimensional systems [51-53]. An explanation was sought of how a two-dimensional Bose liquid, e.g., a thin film of ${ }^{4} \mathrm{He}$, can become superfluid at finite temperature, at odds with the original prediction of Rudolf Peierls and the rigorous proof by Mermin and Wagner that no long-range order associated with Bose-Einstein condensation can occur in two dimensions. The pioneering studies collected under the name of BKT theory, have offered a remedy to that apparent inconsistency by showing that a phase transition is possible from a disordered phase, with an exponentially decaying correlation, to a quasi-long- 
range order with a power-law correlation decay. BKT theory introduced the important concept of topological phase transition and a corresponding critical temperature, above which bound opposite vortex pairs (having zero circulation) unbind, leading to a gas of topological defects formed by single quantized vortices. BKT theory provided a powerful tool for the analysis of phase transitions in a wide range of quasi-two dimensional systems, including superfluid films, cuprates and other more recent hightemperature exotic superconductors, Josephson junction arrays, ultracold atomic gases in light traps, quantum-Hall systems, certain classes of ferroelectrics, etc. It was a tremendous loss for theoretical physics that Vadim Berezinskii passed away prematurely in 1980, at the age of only 45 [54], while BKT theory was triggering an extraordinary advance in the understanding of what we call today topological matter [55]. It was its major protagonist, Duncan Haldane, who was finally associated to Kosterlitz and Thouless for the 2016 Nobel Prize in Physics, disproving the famous Gamow's statement that "only number theory and topology have no application in physics". [55]

About the time of von Klitzing's discovery of the quantum-Hall effect, readily explained by David Thouless, and that of the fractional quantum hall effect by D.C. Tsui, H.L. Stormer, and A.C. Gossard, Duncan Haldane produced as a single author an impressive series of papers on spin chains, low-dimensional magnetic systems and the fractional quantization of the Hall effect [56]. The fractional quantum Hall effect was soon recognized to bring new quantum states of matter allowing for an entirely novel type of topological order. The subsequent introduction of the Berry curvature on the Fermi surface, playing a role similar to that of the Gauss curvature in the Gauss-Bonnet theorem, led Haldane to describe the anomalous Hall effect as a topological property of the Fermi liquid [57]. The evergreen interest in two-dimensional physics has been amplified in recent years by the discoveries of the quantum spinHall effect, the surface conducting states of topological insulators, the evidence of Majorana fermions in condensed matter, graphene, silicene, and various classes of exotic quasi-2D superconductors, including the persistent superconductivity in thin $\mathrm{Pb}$ films down to one single layer.

The deep significance of these advancements is the unified view offered by topology of fundamental physics. Topological insulators are presently a hot topics in condensed matter physics; they owe their name to the special topological structure of the surface energy bands which host electrons of either spin up or spin down and cross the energy gap charac- 
terizing the bulk material. Similarly to the topological robustness described above for graphenes, the topological electronic states are robust under external perturbations, very much like the topology of geometrical figures that (by definition) does not change under continuous deformations. This property, combined with the possibility of spin-up and spindown electrons drifting in opposite directions, allows for stable spin currents and opens new avenues in spintronics and quantum computation. A further help to viable nanotechnologies comes from the very small effective mass, i.e., very large mobility, that electrons have at the intersection of the surface bands of opposite spins (Fig. 10e). Around this point electrons have a small or even null effective mass and the band structure takes the form predicted by the quantum relativistic Dirac equation (see next Section). In this respect there is a similarity between the topological insulators and graphene, where the electron spins are replaced by pseudospins associated with the peculiar conjugated electronic states.

The formal correspondence of electron-hole pairs in condensed matter with particle-antiparticle pairs of Dirac equation is a textbook notion, but symmetry requirements suggest that topological pairs may not be Dirac but Majorana fermions, where particles are their own antiparticles. Thus the long-sought Majorana fermions in neutrino-less double beta decay could finally materialize in condensed matter physics! Frank Wilczek, co-recipient of the 2004 Nobel Prize for his fundamental contributions to quantum chromodynamics, in his 2013 Erice lecture advocated Majorana fermions as a unifying concept in elementary particle and condensed matter physics [58]. On the other hand Ed Witten [46] has been arguing that K-theory in algebraic topology, which has been recently proposed for the classification of topological insulators, is relevant for the classification of D-brane charges in string theory and quantum cosmology. New routes are being opened towards the highly desired grand re-unification of physics under the common paradigm of topology.

\section{Moving a Planck Mass through a Planck Lattice [59]}

There is indeed a wide common ground with a highly productive exchange of ideas, theories and models among particle physics, astrophysics and condensed matter physics. There is however an important caveat: in general quantum field theory in elementary particle physics underlies a continuous uniform space, whereas most condensed 
matter physics refers to crystalline solids with an intrinsic periodicity in space. The important effects arising from the switching on of periodicity can be best understood by considering the transition from gapless to small-gap materials, like graphene.

Graphene actually provides a paradigm of relativistic physics in condensed matter. The lowest electronic conduction band and the highest valence band of a graphene (Fig. 11a) form six double cones with the vertices at the Fermi level (conventionally taken at energy $E=0$ ), so that the Fermi contour is reduces to six equivalent points $\mathrm{K}$ (Dirac points) at the Brillouin zone boundary. The effective mass of Dirac fermions (electrons and holes) at the $\mathrm{K}$ points is zero due to the infinite curvature of the bands at the cone cusps, unlike other electron and hole bands like the ones of energy $E / \gamma_{1}= \pm 1$ at $K$ (Fig. 11a), which have a finite curvature. It should be noted that the Dirac-point degeneracy of the valence and conduction bands is unstable with respect to any modest perturbation, which causes a band splitting at the Fermi level, with a gap opening and a finite effective masses of electrons and holes (Figs. $11 b$ and 12).

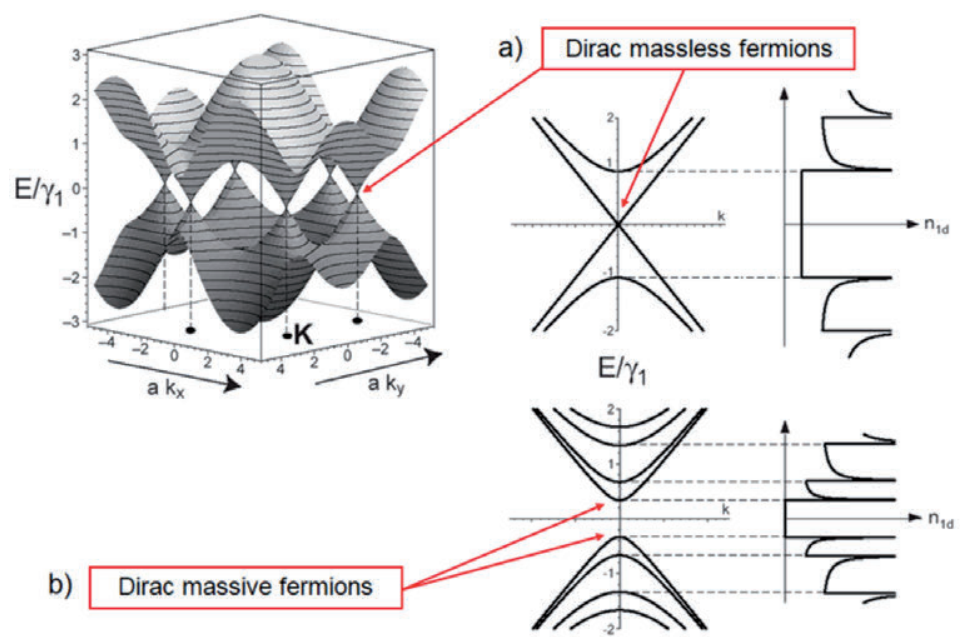

Fig. 11. (a) The lowest electronic conduction band and the bighest valence band of a graphite monolayer (graphene) meet at the Fermi level $(E=0)$ passing through six equivalent points K (Dirac points) at the Brillouin zone boundary (energy in units of the tight-binding coefficient $\gamma_{1}$ ); the effective mass of Dirac fermions (electrons and holes) at the $K$ points is zero due to the infinite curvature of the bands at the cone cusps, unlike other electron and hole bands which have a finite curvature. (b) The Dirac-point degeneracy is unstable with respect to any modest perturbation, which causes a band splitting, with a gap opening and a finite effective masses of electrons and holes. 


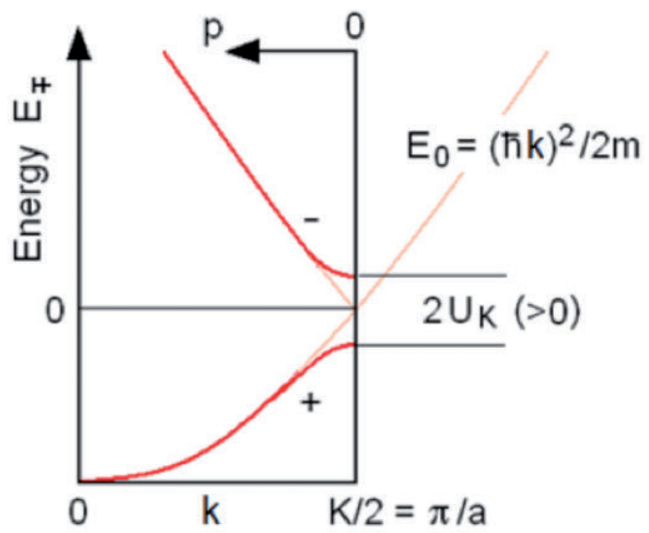

Fig. 12. The effect on a free-particle parabolic band $E_{0}=(\hbar k)^{2} / 2 m$ of a periodic potential, here represented by its Fourier component $U_{K}$ where $K=2 \pi / a$ is the reciprocal lattice vector and a the lattice period. The periodic potential opens a gap at the zone boundary $k=K / 2$ with a splitting of the free-particle band into two bands + and -. Taking the gap midpoint as the origin of the wavevector axis $p=K / 2-k$ and of the energy axis $E=E_{ \pm}(p)$, the bands in the neighborhood of the gap take the relativistic Dirac form.

Fig. 12 illustrates the effects of a periodic potential, here represented by its Fourier component $U_{K}$ where $K=2 \pi / a$ is the reciprocal vector of a lattice of period $a$, on a parabolic free-particle band $E_{0}(k)=(\hbar k)^{2} / 2 m$, where $m$ is the particle mass and $k$ its wavevector. The periodic potential opens a gap at the Brillouin zone boundary $k=K / 2$ (Bragg plane) with a splitting of the free-particle band into two bands+and -. Taking the gap midpoint as the origin of the momentum axis $p=\hbar(K / 2-k)$ and of the energy axis $E=E_{ \pm}(p)$ (with $E=0$ working as the Fermi level), it is a simple solid-state physics exercise to show that the bands in the neighborhood of the gap take the forms:

$E_{\mathrm{m}}(p)= \pm \sqrt{p^{2} c^{2}+m_{\mathrm{m}}^{2} c^{4}}$, where $c \equiv \hbar K / 2 m$

is the lattice Bragg velocity. The reader will immediately recognize Eq. (13) as the relativistic Dirac equation for the particle and anti-particle eigenvalues with the Bragg velocity replacing the speed of light, but there is a subtle difference: the free-particle mass $m$ is replaced by the band effective masses

$$
\frac{m_{\mathrm{m}}}{m}=\frac{1}{\left|1 \pm T_{K} / 2 U_{K}\right|}, \text { where } T_{K} \equiv \frac{(\hbar K)^{2}}{2 m}
$$


is the free-particle kinetic energy corresponding to the momentum $\hbar K$. Since $T_{K}$ is much larger than the gap $2 U_{K}$ (supposed to be small and positive), the two band effective masses are much smaller than the freeparticle mass, and (even more interesting!) they are slightly different with $m_{+}>m$. This is well known to solid state physicists - holes are heavier than electrons -, but it is a serious violation of the particle physics dogma that particles and anti-particles have the same mass. The reason for such a violation is the motion in a periodic potential. Periodicity can be assimilated to a form of quantization or tesselation of space, and the present simple exercise can be easily extended to three dimensions. The idea of space quantization can be traced back to 1899 Planck's invention of units of length and mass

$$
\mathrm{l}_{P} \equiv \sqrt{\frac{h G}{c^{3}}}=4.05 \ldots \cdot 10^{-35} \mathrm{~m}, \quad m_{P} \equiv \sqrt{\frac{h c}{G}}=5.45 \ldots \cdot 10^{-8} \mathrm{~kg}
$$

known as the Planck length and the Planck mass, respectively [60]. They are constructed with the three fundamental constants $c$ (now speed of light!), $G$ (the Newton gravitational constant) and, of course, the brand new Planck constant $h$. Imagine now to have the very big (on the elementary particle scale) Planck mass, $m \equiv m_{P}$, moving in a periodic lattice of period $a \equiv l_{\mathrm{P}} / 2$, which is very, very small on the atomic scale. It turns immediately out that for an effective mass corresponding to that of a proton (which is a factor $10^{-19}$ smaller than the Planck mass), the antiproton-proton mass difference is itself smaller than the proton mass by about the same factor,

$$
\frac{m_{+}-m_{-}}{m_{+}} \cong \frac{2 m_{-}}{m_{P}} \approx 10^{-19},
$$

with the great surprise that

$$
c^{2}\left(m_{-}-m_{+}\right)=-G \frac{m_{-} m_{+}}{a} \text {. }
$$

In words the energy corresponding to the effective mass difference is equal to the gravitational potential between particle and antiparticle at the lattice distance! This interesting result can be interpreted by saying that gravity is a quantum effect due to the motion on a periodic lattice. The periodic potential responsible for the gap implies a perturbing field which provides particles and anti-particles with a finite mass. This doesn't sound new, whereas the unexpected fact is that this field also provides the gravitational interaction between them. After all 
also this is due, because the process in which a neutral boson annihilates into a particle-antiparticle pair has to provide not only their mass energy (the gap) and their kinetic energy, but also their gravitational energy at the shortest possible distance, the Planck lattice distance. Thus the gravitational potential at any distance $r$ can also be expressed in terms of the absolute effective mass difference:

$V_{G}(r)=-\frac{|\Delta m|}{2 m_{P}} \frac{h c}{r}$,

with the Planck's constant signalling the quantum nature of the gravitational potential. One could speculate that Newton gravitation originates from the quantization of space, but for a condensed matter physicist there is a more concrete and practical question: what does this mass-difference energy mean in the original system where $a$ is the lattice distance and $c$ the Bragg velocity? A natural system is a semiconductor, e.g., germanium, where the difference between hole and electrons effective masses is of the order of one tenth of the electron mass $m$. In this case what we will call a pseudo-gravitational potential is given by

$$
V_{e h}(r)=-\frac{h^{2}}{4 a m^{2}} \frac{m_{h}-m_{e}}{r} .
$$

At a distance of the order of the lattice parameter of Ge this potential is of the order of $0.1 \mathrm{eV}$, which is neither large nor small as compared to the gap or to the optical phonon energies. A direct meaning of this energy is found by considering the Rydberg for the exciton (electron-hole bound state) hydrogenic ground state. The difference in the Rydberg energy produced by the difference in mass,

$$
\begin{aligned}
R y_{e h}-R y_{e h}^{0} & \cong \frac{e^{4}}{8 \hbar^{2} \varepsilon_{s}^{2}}\left(m_{e}-m_{h}\right)=-\frac{e^{4}}{4 \hbar^{2} m \varepsilon_{s}^{2}} m_{e} m_{h} \\
& =-\frac{e^{2}}{4 m^{2} \varepsilon_{s}^{2}} \frac{m_{e} m_{h}}{a_{0}},
\end{aligned}
$$

where $e$ is the elementary charge, $e_{s}$ is the dielectric constant of germanium, and $a_{0}$ the exciton Bohr radius, also takes the form of an attractive gravitational potential with an effective constant " $G$ " $\equiv e^{2} / 4 m^{2} \varepsilon_{s}^{2}$. A closer inspection would show that this energy corresponds to the difference between the exciton energy as referred to its center of mass and that referred to the crystal center of mass. 


\section{CONCLUSIONS}

Graphenes in all their forms, as well as many condensed matter systems where topology plays in some general aspects a major role as compared to other local and metric properties, can provide an all-purpose quantum construction kit. They can simulate at the small scale of a desk-top laboratory (where experiments are comparatively easy to design for a given purpose) situations of great relevance in quantum theory that occur in other domain of physics, however over very different scales of space and time not easily accesible to experiment. A few examples concerning particle physics or astrophysics have been briefly mentioned in Section 4. The main message that can be extracted from the few points discussed above, as well as from the fashinating topics which have been illustrated in all lectures presented at this meeting on the modern triathlon "heat-electricity-mechanics", is that there is nevertheless one physics, and that a grand-reunification of largely separated branches of physics needs to be pursued under some general flags like topological quantum field theory.

\section{REFERENCES}

[1] K. S. Novoselov, A. K. Geim, S. V. Morozov, D. Jiang, Y. Zhang, S. V. Dubonos, I. V. Grigorieva, A. A. Firsov, Science 30, 666 (2004).

[2] K. S. Novoselov, A. K. Geim, S. V. Morozov, D. Jiang1, M. I. Katsnelson, I. V. Grigorieva, S. V. Dubonos, and A. Firsov, Nature, 438, 197 (2005)

[3] A. H. Castro Neto, F. Guinea, N. M. R. Peres, K. S. Novoselov, and A. K. Geim, Rev. Mod. Phys. 81,109 (2009).

[4] G. Benedek, M. Bernasconi, E. Cinquanta, L. D'Alessio, and M. De Corato, The Topological Background of Schwarzite Physics, in Mathematics and Topology of Fullerenes, edited by F. Cataldo, A. Graovac and O. Ori, Springer Ser. on Carbon Materials Chemistry and Physics, Vol. 4 (Springer, Heidelberg Berlin 2011), Chap. 12

[5] H. P. Boehm, A. Clauss, G. O. Fischer, U. Hofmann, Zeitschr.Anorg. Allgem. Chem. 316, 119 (1962).

[6] W. M. Hess and L. L. Ban, Norelco Reporter 13 (1966) 102; and Proc. 6th Int. Congr. on Electron Microscopy (Kyoto) vol 1, p 569

[7] A.M. Shikin. D. Farìas, and K.H. Rieder, Europhys. Lett. 44, 44 (1988).

[8] T. Aizawa, R. Souda, S. Otani, Y. Ishizawa, C. Oshima, Phys. Rev. B 42 (1990) 11469, and 43, 12060 (1991) (E).

[9] H. W. Kroto, J. R. Heath, S. C. O'Brien, R. F. Curl and R. E. Smalley, Nature 318 , $162(1985)$ 
[10] H.W. Kroto, J.E. Fischer, and D. Cox, The Fullerenes, 1st ed. (Pergamon, 1993).

[11] S. Iijima, Nature 324, 56 (1991); M. Endo, S. Iijima, M.S. Dresselhaus, Carbon Nanotubes, (Elsevier, 2013)

[12] A. L. McKay, Nature 314, 604 (1985); A. L. McKay and H. Terrones, Nature 352, 762 (1991).

[13] M. O'Keeffe, G. B. Adams and O. F. Sankey, Phys. Rev. Lett. 68, 2325 (1992).

[14] D. Donadio, L. Colombo, P. Milani and G. Benedek, Phys. Rev. Lett., 84, 776 (1999).

[15] E. Barborini, P. Piseri, P. Milani, G. Benedek, C. Ducati, and J. Robertson Appl. Phys. Lett. 81, 3359 (2002); highlighted by Ed Gerstner, Nature, Materials Update (7 Nov. 2002), http://wwwnaturecom/materials/news/news/021107/ portal/m021107-1html

[16] Benedek G, Milani P, and Ralchenko VG (eds), Nanostructured carbon for advance applications (Kluwer, Dordrecht 2001).

[17] G. Benedek, H. Vahedi-Tafreshi, E. Barborini, P. Piseri, P. Milani, C. Ducati C, and J. Robertson, Diamond Relat. Mater. 12, 768 (2003).

[18] H. A. Schwarz, Gesammelte Mathematische Abhandlungen, 11 (Springer, Berlin 1990)

[19] E. Heckel, Report on the Radiolaria collected by H. M. S. Challenger during the Years 1873-1876 (Eyre \& Spottiswoode, London 1887) Plate 111, p. 1634.

[20] A. C. Ferrari, B. S. Satyanarayana, J. Robertson, W. I. Milne, E. Barborini, P. Piseri, and P. Milani, Europhys. Lett. 46, 245 (1999).

[21] I. Boscolo, P. Milani, M. Parisotto, G. Benedek, and F. Tazzioli, J. Appl. Phys. 87, 4005 (2000).

[22] Z. Qin, G. S. Jung, M. J. Kang, and M. J. Buehler, Qin et al. Sci. Adv. 3, e1601536 (2017)

[23] G. Benedek, H. Vahedi-Tafreshi, P. Milani and A. Podestà, "Fractal Growth of Carbon Schwarzites" in Complexity, Metastability and Non-Extensivity, edited by C. Beck et al. (World Scientific, Singapore 2005), p. 146-155.

[24] W. Helfrich, Z. Naturforsch. 28, 768 (1973).

[25] S. T. Hyde, in Foams and Emulsions, J. F. Sadoc and N. Rivier (Kluwer, Dordrecht, 1999) p. 437.

[26] C. Oguey, in Foams and Emulsions, J. F. Sadoc and N. Rivier (Kluwer, Dordrecht, 1999) p. 471.

[27] J. M. Sullivan, in Foams and Emulsions, J. F. Sadoc and N. Rivier (Kluwer, Dordrecht, 1999) p. 379.

[28] C. T. White et al, in Buckminsterfullerenes, W. E. Billups and M. A. Ciufolini (VCH, New York, 1993) p. 125.

[29] S. Gaito, L. Colombo and G. Benedek, Europhys. Lett. 44, 525 (1998).

[30] M. de Corato, M. Bernasconi, L. d'Alessio, O. Ori, and G. Benedek, Topological versus Physical and Chemical Properties of Negatively Curved Carbon Surfaces in Topological Modeling of Nanostructures and Extended Systems. ed. by A. R. Ashrafi, F. Cataldo, A. Iranmanesh, and O. Ori, Springer Series in Carbon Materials: Chemistry and Physics, Vol. 7, Chap. 4 (Springer, Heidelberg Berlin 2013) pp 105-136.

[31] M. Bühl and A. Hirsch, Chem. Rev. 101, 1153 (2001) 
[32] M. De Corato and G. Benedek, in Epioptics 11, ed. by A. Cricenti (World Sci., Singapore 2012) p. 114-131; ISBN 978-981-4417-11-2

[33] R. L. Cappelletti, J.R.D Copley, W.A. Kamitakahara, F. Li, J.S.Lannin, and D. Ramage, Phys. Rev. Lett. 66, 3261 (1991).

[34] J. I. Gersten and F. W. Smith, The Physics and Chemistry of Materials (Wiley \& Sons, Inc, New York 2001) p. 176

[35] V. Rosato, M. Celino, S. Gaito and G. Benedek, Phys. Rev. B 60, 16928 (1999)

[36] K. V. Zakharchenko, A. Fasolino, J. H. Los, and M. I. Katsnelson, J. Phys. Cond. Mat. 23, 202202 (2011)

[37] H. Wiener, J Am Chem Soc 1, 17 (1947).

[38] O. Ori, F. Cataldo, and A. Graovac, Fuller. Nanotub. Carbon Nanostruct. 17, 308-323 (2009)

[39] The following paragraphs report some of the concepts exposed in the article: G. Benedek, When Topology Matters: The Nobel Prize in Physics 2016, Revue des Questions Scientifiques 188 (2) 1-7 (2017)

[40] G. Van der Mensbrugghe, "Notice sur Joseph Antoine Ferdinand Plateau", Annuaire Académie Royale de Belgique, LI, 389-486 (1885).

[41] J. E. Taylor, Annals of Mathematics. Second Series, 103, 489-539 (1976).

[42] Lord Kelvin (Sir William Thomson), Phil. Mag., 24, 503 (1887) .

[43] D. Weaire and R. Phelan, Phil. Mag. Lett., 69, 107 (1994)

[44] J. L. Bell, and H. Korté, Hermann Weyl, in The Stanford Encyclopedia of Philosophy, E. N. Zalta (ed.), (Winter 2016 Edition) https://plato.stanford.edu/archives/win2016/entries/weyl/

[45] See, e.g., K. Hashimoto, "D-Brane: Superstrings and New Perspective of Our World" (Springer, 2012)

[46] E. Witten, J. High Energy Phys. 12, 012 (1998); ibidem 12, 012 (1998).

[47] G. Galvani, et al, Phys. Rev. Letters 77, 3573 (1996)).

[48] A. J. Hanson, Notices of the Amer.Math.Soc.41,1156 (1994)

[49] C. McAllen, Exact mathematical plot of a Lorentzian wormbole 2007 (from Wikipedia Commons) (Schwarzschild wormbole)

[50] S. H. Yao, et al, in Focus Issue onTopological Insulators - From Materials Design to Reality, ed. by Claudia Felser, Shoucheng Zhang, and Binghai Yan, Phys. Status Solidi RRL 1, 2 (2013).

[51] V. L. Berezinskii, Zh. Eksp. Teor. Fiz. 61, 1144 (1971); Sov. Phys. JETP 34, 610 (1972).

[52] J. M. Kosterlitz and D. J. Thouless, J. Phys. C 6, 1181 (1973).

[53] J. V. José (ed.), "40 Years of Berezinskii-Kosterlitz-Thouless Theory" (World Scientific Publ. Co., Singapore 2013).

[54] A. A. Abrikosov, L. P. Gor'kov, I. E. Dzyaloshinski, A. I. Larkin, A. B. Migdal, L. P. Pitaevski, and I. M. Khalatnikov, "Vadim L'vovich Berezinski" Sov. Phys. Usp., 24, 249 (1981).

[55] See the Focus in Nature Phys., 12, 615-718 (2016).

[56] F. D. M. Haldane, Phys. Rev. Lett. 50, 1153 (1983); Phys. Lett. A 93, 464 (1983); Phys. Rev. Lett. 51, 605 (1983).

[57] F. D. M. Haldane, Phys. Rev. Lett. 93, 206602 (2004).

[58] F. Wilczek, Majorana returns, Nature Phys. 5, 614 (2009); Wilczek's lecture was 
delivered at the 59th Course "Majorana Physics in Condensed Matter" of the Int. School of Solid State Physics, 12-18 July 2013, at the Ettore Majorana Foundation and Centre for Scientific Culture, Erice, Sicily. Directors of the Course: X. Chen. R. Fazio, J. Moore, V. Pellegrini, and M. Polini.

[59] First presented in the occasion of the 2009 opening lecture at the PhD School in Physics of the University of Pavia.

[60] M. Planck, Sitzingsber. Königl. Preuss. Akad. Wissensch. Berlin 5, 440 (1899); here it is convenient to adopt the definition with the original Planck's constant $b$ rather than the reduced Planck's constant $\hbar=b / 2 \pi$. 